from parent to offspring. The innumerable variations however? Not so much. They are, after all, innumerable.

That does not mean that science is not trying, and on pages 68 and 75 of this issue, Nature publishes the latest progress reports from this colossal effort. The papers mark the completion of the 1000 Genomes Project, the largest work yet to sequence the genetic information of hundreds of individuals in an attempt to tune into Mother Nature's hum of human variation. It completes a set of genomic reference tools - resources of genetic data produced by international collaborations - that dates back 25 years to the start of the Human Genome Project.

The bigger job, of tracking the relationships between genetic variation and human disease to help to develop effective treatments, is not finished, and may never be. But it is important from time to time to acknowledge and celebrate landmarks of achievement along the way. This week marks one such landmark.

The data sets produced by the 1000 Genomes Project are already in use. The genetic details of the volunteers provide a publicly owned and openly available asset in the era of big data, and offer a foundation for further study. Applications range from hunts for the genetic roots of human illness to analyses of population genetics and evolutionary history.

As technology continues to improve, so does the ability to capture genetic variation worldwide. The research published this week demonstrates that neatly. For a start, the eponymous 1,000 genomes analysed have extended to more than 2,500 . The data now come from 2,504 individuals, across 26 distinct populations. From Chinese immigrants in downtown Denver, Colorado, and the Luhya tribe in Kenya to Punjabis in the dusty streets of Lahore, Pakistan, much of human life and diversity is here. The genetic data have been analysed more thoroughly than was possible before, which throws more light on rarer forms of variation.

The take home message: although most common genetic variants are shared across populations, rarer variants are often restricted to closely related groups. Many more rare variants are still to be identified.

The improved precision provided in this latest data set has also

enabled a more comprehensive map of structural variation across the human genome. For the first time, this includes analysis of eight structural-variation classes.

What now? Sequencing projects should continue to cast the net wide, and extend it further, to seek volunteers from regional and ethnic groups that are currently under-represented in global genetic databases. Meanwhile, the astonishing increase in genetic sequenc-

"The final goal remains to make this flood of population-level genetic research relevant to

personal health." ing ability - even when compared with when the 1000 Genomes Project began in 2007 - has shifted the research bottleneck from generation of data to analysis and interpretation. Two challenges are to make sense of the non-coding regions of DNA and to tease out the links between genetic variation and clinical symptoms.

To exploit the gathered genetic information, more projects need to link and cross-reference it to clinical information and well-characterized phenotype data sets. On page 82 , the UK10K Consortium publishes an early example of the latter: the first large-scale demonstration of whole-genome sequencing linked to complex traits.

As links to health records are established - and some, such as the UK Biobank study and the US Precision Medicine Initiative, are already on the books - it is crucial that public trust is secured. The ways in which scientists collect, store and share sensitive personal information must continue to evolve to ensure adequate safeguards. The Global Alliance for Genomics and Health has offered promising alternatives and a model to follow.

The final goal remains to make this flood of population-level genetic research relevant to personal health. Emerson would have approved. He was a proponent of individualism, a political philosophy that emphasizes the moral worth of the individual. He celebrated the non-conformist. And when it comes to the few laws that dictate the repetition of genetics, it is not just the 2,504 people whose variation is detailed this week who are the non-conformists. We all are.

\section{Goals galore}

\section{The latest global targets from the United Nations must be translated into realistic policies.}

\section{$\mathrm{T}$} The nations of the world approved a new development agenda in New York over the past weekend. The United Nations' 17 Sustainable Development Goals cover topics ranging from poverty reduction to environmental sustainability, and are accompanied by 169 detailed targets that are intended to help governments and aid organizations to focus resources. It is a noble initiative, in principle, and the world would undoubtedly be a better place by its target year of 2030 if these goals were met. But despite the promotional efforts - one of the main side events over the weekend culminated in pop diva Beyoncé and the rock band Pearl Jam performing Bob Marley's 'Redemption song' in New York's Central Park - it remains unclear what impact the goals will have on global affairs.

One problem is that there is a sense of déjà vu here. Back in 1992, the world set out a 351-page manifesto for human justice and environmental sustainability at the Earth Summit in Rio de Janeiro, Brazil. Eight years later, the UN adopted the eight Millennium Development Goals, which included halving extreme poverty rates and achieving universal primary education by 2015 .

The aspirational agenda is still clear, but so too are the barriers to investment - they include corruption, political instability, poor education systems, malfunctioning regulatory systems and the lack of a skilled workforce.

The real challenge is to identify and implement realistic policies that will get us where we say we want to be, and this is where academics must engage. The next step for the Sustainable Development Goals is to identify a range of health, economic and environmental indicators that can be used to track progress.

That debate is expected to extend into next year, and researchers should work to ensure that governments are collecting and reporting data. Scientists and policymakers must also redouble efforts to identify effective - and politically viable - strategies in which to invest a limited supply of money. Increasingly, development economists are doing just that, complete with rigorous testing, but there is scope for much more research in this important field.

The first and perhaps biggest opportunity to address some of these issues in a significant way will come when global leaders converge on Paris for the UN climate summit this December. Attempts to develop a telling international climate regime have languished for a quarter of a century, but there are signs of life, and governments around the world - rich and poor alike - are beginning to engage. The world is unlikely to see a single solution emerge, but the summit could produce a framework that will push all governments to invest in the policies, as well as in the science and technology.
DNATURE.COM To comment online, click on Editorials at: go.nature.com/xhunqv
Trillions of dollars of investment over the coming decades, public and private, are on the table. Directing that money to the right technologies and the right places would go a long way towards improving lives. 